\title{
Complement Genes in the Asterids Comparisons with the Echinids (Echinoderma)
}

\author{
Michel Leclerc \\ Immunologie Des Invertébrés Université d’Orléans 45100 Orléans Cedex 2, France
}

Received 2013-03-11, Revised 2013-04-17; Accepted 2013-05-06

\begin{abstract}
The axial organ of the sea star Asterias rubens (Asterid) is a primitive immune organ. The B-like cells, when stimulated by various antigens, produce antibody substances correlating with ig kappa genes. On the other hand, component complement genes were found. For each component, one or several contigs were analysed. It is said that Asterias forbesi, another sea-star, in earlier results, showed complementlike activity. A brief comparison with the complement system in sea urchin (Echinid) was performed, especially about the $\mathrm{C} 3$ component.
\end{abstract}

Keywords: Echinid, Asterias Rubens, Component, Echinoderma

\section{INTRODUCTION}

A large number of investigations performed in the last few years, in our Laboratory, have provided evidence that the sea star Asterias rubens (echinoderma) possesses a primitive immune system with cellular and humoral responses functionally similar to those of the immune system of vertebrates.

When isolated axial organ cells were stimulated "in vitro" by hapten-carriers, a soluble factor was secreted in the culture medium, that specifically lysed sheep red cells sensitived with the corresponding antigen (Brillouet et al., 1986). This lytic reaction required the presence of thermolabile factor (complement) present in mammal serum and in the coelomic fluid of the seastar. Moreover, it could be demonstrated that this antibody factor (Delmotte et al., 1986), « unknown » in the sea urchin system (Hibino et al., 2006) was produced by the B-like cells but only, when T-like and phagocytic cells were present during the antigenic stimulation (Leclerc et al., 1986).

We have now observed that the antibody factor was correlated with IgKappa genes (Leclerc et al., 2011) On the other hand, complement genes were found. In earlier studies (Leonard et al., 1990) in Asterias forbesi, another sea star, complement-like activity was shown.

\section{MATERIALS AND METHODS}

Sea stars Asterias rubens were obtained from the Biology Institute (Gothenburg University). Immunizations were performed on 20 animals, in an aquarium with sea water at $10^{\circ} \mathrm{C}$ by using Horse-Radish Peroxydase (HRP) (Sigma Products) as antigen at a concentration of $1 \mathrm{mg}$ $\mathrm{mL}^{-1} .20$ non-injected animals were used as controls. The axial organs were removed; RNA was extracted, using Trizol (Invitrogen) according to manufacturer instructions, from immunized sea stars (HRP) and Controls (C). cDNA was normalized using double strand specific nuclease essentially as described by Zhulidov et al. (2004). cDNA was fragmented using DNA Fragmentase (New England Biolabs), according to the manufacturer's instructions. After ligation of adapters for illumina's GSII sequencing system, the cDNA was sequenced on the illumina GSII platform sequencing $1 \times 100 \mathrm{bp}$ from one side of the approximately $200 \mathrm{bp}$ fragments. Sequences were assembled using Velvet (Zerbino and Birney, 2008). Assembled nodes were used for further assembly including Beta vulgaris EST-Data from NCBI in MIRA.

\section{RESULTS AND DISCUSSION}

First, Components of the classical pathway as compared to mammals, are given with: 


\subsection{Control-Complement C1q Subcomponent Subunit A}

One contig (NODE_55223_length_184_cov_8.456522) could be annotated via BLASTX to mouse "Complement C1q subcomponent subunit A" from the SWISSPROT database, had an e-value of $8.55742 \mathrm{e}-06$. On an aligned region of 72 amino acids, 29 positive and 25 identical amino acids were found.

\subsection{HRP-Complement C1q Subcomponent Subunit A}

No contig matched, no hit was found. Then,

\subsection{Control-Complement C1q Subcomponent Subunit B}

One

contig

(NODE 48557 length 161 cov 19.316771) could be annotated via BLASTX to mouse "Complement C1q subcomponent subunit B" from the SWISSPROT database, had an e-value of $8.55742 \mathrm{e}-06$. On an aligned region of 57 amino acids, 33 positive and 19 identical amino acids were found.

\subsection{HRP-Complement C1q Subcomponent Subunit B}

One contig (NODE_68235_length_178_cov_7.724719) could be annotated via BLASTX to mouse "Complement C1q subcomponent subunit B" from the SWISSPROT database, had an e-value of 3.97232e-10. On an aligned region of 44 amino acids, 39 positive and 28 identical amino acids were found then.

\subsection{Control-Complement C1q Subcomponent Subunit C}

One

NODE 37343 length 278 cov 12 356115) contig aligned region of 39 amino acids, 25 positive and 19 identical amino acids were found.

\subsection{HRP-Complement C1q Subcomponent Subunit C}

Three contigs could be annotated via BLASTX to mouse "Complement C1q subcomponent subunit C" from the SWISSPROT database. The best contig (NODE_29181_length_277_cov_7.790614) had an e-value of 3.67148e-09. On an aligned region of 45 amino acids, 27 positive and 22 identical amino acids were found.

\subsection{We Observe Now the C2 Component}

Five contigs could be annotated via BLASTX to mouse "Complement C2" from the SWISSPROT database. The best contig (NODE_23089 length_519_cov_15.136802) had an evalue of $7.65302 \mathrm{e}-07$. On an aligned region of 153 amino acids, 59 positive and 38 identical amino acids were found. And now what about the component C4?

\subsection{Control - Complement C4-B}

One contig (NODE_95392_length_215_cov_10.818604) could be annotated via BLASTX to mouse "Complement C4-B" from the SWISSPROT database, had an e-value of 3.48532e-06. On an aligned region of 76 amino acids, 40 positive and 21 identical amino acids were found.

\subsection{HRP-Complement C4-B}

One contig (NODE_58696_length_164_cov_11.884147) could be annotated via BLASTX $\bar{X}$ to mouse "Complement C4-B" from the SWISSPROT database, had an e-value of $7.51259 \mathrm{e}-05$. On an aligned region of 44 amino acids, 26 positive and 21 identical amino acids were found.

Then, we have a look about the $\mathrm{C} 3$ component which is central, in mammals, to both the classical and alternative pathways. We try, at the end of this chapter to attempt a comparison with the $\mathrm{SpC} 3$ of the sea urchin.

Seven contigs could be annotated via BLASTX to mouse "Complement C3" from the SWISSPROT database. The best contig (NODE_25262_length_1200_cov_21.958334) had an e-value of $3.2395 \mathrm{e}-49$. On an aligned region of 355 amino acids, 128 positive and 173 identical amino acids were found.

\subsection{HRP-Complement C3}

Eleven contigs could be annotated via BLASTX to mouse "Complement C3" from the SWISSPROT database. The best contig (NODE_15219_length_398_cov_19.402010) had an evalue of $4.96 \overline{748 \mathrm{e}-24 .}$. On an aligned region of 146 amino acids, 79 positive and 59 identical amino acids were found. It must be noted that $\mathrm{C} 3$ belongs to, also, the ALTERNATE pathway.

Comparisons with the sea urchin (Strongylocentrotus purpuratus) Fig. 1:

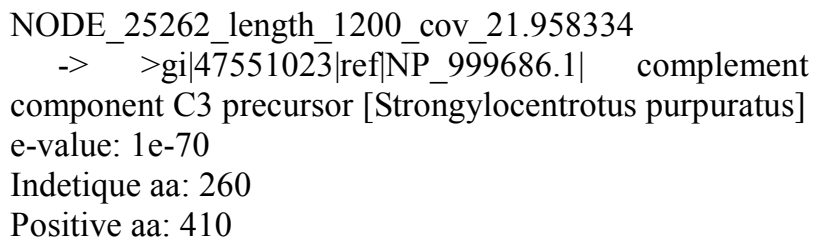
component $\mathrm{C} 3$ precursor [Strongylocentrotus purpuratus] e-value: 1e-70

Indetique aa: 260

Positive aa: 410 


\begin{abstract}
TCACTGTCTGGCTCTGGGATGTGGTAGCTTGACTCAAACTTCAGCTGGCCAACTCCTGTC CCTGAAGAGTCAAAATGAACACGGAATTCCGGCTCATTTCCTGGAATCACAAGTTTAGA GTGCTGTCTGACGATTGCGTTGAACGGTTGCAGAACGAACTCATCTTCAAATTGACTCTC AATACAACTGACCTGGCAATTGATATCAATGTTGCTCGTCTCGGACTTGATAGCATACTCC GACAACGCCTGCAGAGCTATAACTGTATCCTGAGAAGACACAAATCCACCTTCATAATTC TCTTGTTCCGTGAGCCAATTAACAATGGCATGACTGTAGGGCAGGTCGTCCAGCTGAAGT AAAGCCAGCAGTGCATAGCTTGTCATCTCTACATCAATGGCTTTCGGCCGGTTGATGTAC CAATATGGTTTCGGACCCGCGCCAAAGGACGAGTCGTCTGCTCCCCAGTGACGATAATTG GTTGCTCTATCGTACGTAGCGATGTCTTTGAGCATCGTGAGAGCTTCATCAGCTTTGTTGC TATTGGCCAGAGCGAGGGCGTATGCGGTGATGGCAATGGCGTAAGGTCTCGTCAATTGT TGCAGTTGACCCTCTAAGAAAGCCGTAGCTGAAGCAACAGAGTCAGTCTTACTTACTGTT TCACATTCACACTCCAGAAGAGCTATCAGCACGTATGCAGTAAGCGAGGCGTCACCTTGC CACCACCAATCATTTCTTGGTGATGCACTTTGTACAATTCCCCGAACGCACCGTTGTCAAA TTCTGTGTCTCCTCTATCAGCCATCTCATAGCATTACAGGTGATGCTTCCATCAATGTAGG CAAATTCTTTGGCCTGGCAGAACACCTTGCTGACAAAGGCCGTCAACCAGGTACTGCTTG GATACTGTAAATTATCTCCCCATACTGAAAATGAACCGTCCGAACGACGATGCGTCAATT TCTTGAGTTATACCGCCTCCGATGTACTGCTGAGCGGACACCTCCAGCTCAGCCGTGAAC TGATTAGTGTGCTTCAGATACCGGTAGACGTAGACGTTGGGTGCTAGGGTGATCATCGTT TGTTCACCACACCCTCGAGGGATCTGCAAAATGTCGCCCAACCCACTGATGACCGTGTCG ATGACGTTACCCAAAATATTTCCCATGATGCTGATATGGGTTTGATGGGTACCAGGGATA GCGTCACTATGCAAGGAGTATCGT
\end{abstract}

Fig. 1. DNA sequence of $\mathrm{C} 3$ component (comparisons with S. purpuratus. Echinid)

>NODE_25262_length_1200_cov_21.958334

We give now the obtained results concerning the components of the membrane-attack complex as compared to mammals. So with the C9 component.

\subsection{Control-Complement Component C9}

Three contigs could be annotated via BLASTX to mouse "Complement component C9" from the SWISSPROT database. The best contig (NODE_46472_length_143_cov_10.104895) had an evalue of $1.417 \overline{4} 6 \mathrm{e}-05$. On an aligned region of 31 amino acids, 17 positive and 15 identical amino acids were found.

\subsection{HRP-Complement Component C9}

3.12.1. No Contig Matched, no hit was Found. As for the C5 Component

\subsubsection{Control-Complement C5}

One contig (NODE_75605_length_236_cov_32.677967) could be annotated via BLASTX to mouse "Complement C5" from the SWISSPROT database, with an e-value of 0.000634854 . On an aligned region of 55 amino acids, 27 positive and 19 identical amino acids were found.

\subsection{HRP-Complement C5}

Two contigs could be annotated via BLASTX to mouse "Complement C5" from the SWISSPROT database. The best contig (NODE_7717_length_665_cov_13.831579) had an e-value of $6.42528 \mathrm{e}-17$. On an aligned region of 212 amino acids, 99 positive and 57 identical amino acids were found. At last we observe the C8 Component and we find:

\subsection{Control-Complement Component C8 Alpha Chain}

Twelve contigs could be annotated via BLASTX to mouse "Complement component $\mathrm{C} 8$ alpha chain" from the SWISSPROT database. The best contig (NODE 68667 length 311_cov_10.845659) had an evalue of $6.799 \overline{5} \mathrm{e}-15$. $\overline{\text { On }}$ an aligned region of 95 amino acids, 33 positive and 42 identical amino acids were found.

\subsection{HRP-Complement Component C8 Alpha Chain}

Six contigs could be annotated via BLASTX to mouse "Complement component C8 alpha chain" from the SWISSPROT database. The best contig (NODE_43169_length_2163_cov_11.209893) had an evalue of $6.36046 \mathrm{e}-14$. On an aligned region of 45 amino acids, 42 positive and 33 identical amino acids were found.

\section{CONCLUSION}

The main components of Complement as compared to mammal ones were found in the sea star: How genes are expressed? By which pathway? In the present time we have no answers to these questions.

It could be a working hypothesis which needs to be explored. Nevertheless these observations indicate that 
certain mammal structures are present in the immune system of the sea star: and this finding strongly supports the idea that an effective immune system is already present in Echinoderma.

Since the typical immune system is not present in invertebrates, it can be suggested that it was developed at this point in evolution. The main acquisition of Asterids seems to be the cellular differentiation in two subpopulations of cells, ancestral to $T$ and $B$ lymphocytes and their interplay with phagocytes resulting in the synthesis of specific humoral antibody factor (Delmotte et al., 1986) in relation with the Complement to be expressed.

Alternative and direct pathway genes correlated with « mammal » genes were discovered in this study, at the difference of another Echinoderma: the sea-urchin: Strongylocentrotus purpuratus (Smith et al., 2001) which shows only two components of the complement such as a homologue of $\mathrm{C} 3$, called $\mathrm{SpC} 3$, having similarities with sea star C3 component (Fig. 1) and factor Bf possessing significant similarity to the vertebrate $\mathrm{Bf} / \mathrm{C} 2$ family .No humoral immune reaction occur in the sea urchin at our knowledge. At last, It could be expected that the sea-star specific immune reaction needs all the genes (7 components) from $\mathrm{C} 1$ to $\mathrm{C} 9$ to be expressed. This study reveals a true progress in the comprehension of the immune system in Echinoderma.

\section{REFERENCES}

Brillouet, C., M. Leclerc and G. Luquet, 1986. Mitogens induced regulation of sea star axial organ cell humoral immune response in vitro. Cell Biol. Int. Rep., 10: 667-675. DOI: 10.1016/03091651(86)90144-X
Delmotte, F., C. Brillouet, M. Leclerc, G. Luquet and J.C. Kader, 1986. Purification of an antibody-like protein from the sea star Asterias rubens (L.). Eur. J. Immunol., 16: 1325-1330. PMID: 3096739

Hibino, T., M. Loza-Coll, C. Messier, A.J. Majeske and A.H. Cohen et al., 2006. The immune gene repertoire encoded in the purple sea urchin genome. Dev. Biol., 300: 349-365. PMID: 17027739

Leclerc, M., C. Brillouet, G. Luquet and R.A. Binaghi, 1986. The immune system of invertebrates: The sea star Asterias rubens (Echinoderma) as a model of study. Bull. Inst. Pasteur, 84: 311-330.

Leclerc, M., S. Dupont, O. Ortega-Martinez, B. Hernroth and N. Krezdorn et al., 2011. Evidence of Kappa genes in the sea-star Asterias rubens (Echinoderma). Immun. Lett., 138: 197-198. DOI: 10.1016/j.imlet.2011.01.016

Leonard, L.A., J.D. Strandberg and J.A. Winkelstein, 1990. Complement-like activity in the sea star, Asterias forbesi. Dev. Comput. Immunol., 14: 19-30. PMID: 2338154

Smith, L.C., L.A. Clow and D.P. Terwilliger, 2001. The ancestral complement system in sea urchins. Immunol. Rev., 180: 16-34. PMID: 11414357

Zerbino, D.R. and E. Birney, 2008. Velvet: Algorithms for de novo short read assembly using de Bruijn graphs. Genome Res., 18: 821-829. DOI: 10.1101/gr.074492.107

Zhulidov, P.A., E.A. Bogdanova, A.S. Shcheglov, L.L. Vagner and G.L. Khaspekov et al., 2004. Simple cDNA normalization using kamchatka crab duplexspecific nuclease. Nucl. Acids Res., 32: e37-e37. PMID: 14973331 